\title{
SUPERVIVENCIA Y CRECIMIENTO INICIAL DE SUBESPECIES Y PROCEDENCIAS DE Acacia saligna (Labill.) H.L. WendI EN EL SECANO DE LA REGIÓN DEL MAULE, CHILE
}

\author{
Gutiérrez, Braulio ; Rojas, Patricio ${ }^{8}$ y Soto, Hernán ${ }^{6}$
}

\section{RESUMEN}

Acacia saligna es cultivada como especie exótica en la Región de Coquimbo, donde existen alrededor de 7.500 ha orientadas principalmente a producción de forraje, combustible, recuperación de suelos y control de erosión. Sus semillas además poseen aplicaciones potenciales en la industria de alimentos funcionales. La gran variabilidad exhibida por la especie sugiere que también podría cultivarse en el secano costero e interior de la Región del Maule.

A pesar de lo anterior, su baja digestibilidad y altos contenidos de taninos hacen de $A$. saligna un forraje poco atractivo como fuente única de alimento para ganado. Sin embargo, esta característica, así como su crecimiento, supervivencia y otras pueden ser objeto de mejora al explorar adecuadamente la enorme variabilidad que la especie presenta.

En este contexto, en mayo de 2018 se evaluó dos ensayos de procedencias con el objetivo de examinar su supervivencia y crecimiento, uno en la zona de transición costa-secano del Maule (Chanco) establecido en el año 2014 y otro en el secano interior de la misma región (Cauquenes), establecido el año 2015. Estos ensayos contienen una colección de 11 procedencias de Western Australia, que incluye a las principales cuatro subespecies reconocidas para esta especie: lindleyi, saligna, pruinescens y stolonifera. Antes de establecer estos ensayos, en el país solo habría estado presente la subespecie pruinescens con una pequeña participación de lindleyi en otros ensayos del Instituto Forestal en la Región de Coquimbo.

Mediante análisis de varianza y comparaciones múltiples de medias se concluye que en la zona de transición costa-secano (Chanco) las mejores procedencias, en términos de crecimiento y supervivencia, corresponden a Bambun Rd. y Palmer Block, mientras que las mejores subespecies resultan ser pruinescens y saligna; por su parte, en el secano interior (Cauquenes) las mejores procedencias en cuanto a supervivencia y crecimiento fueron Flynn Drive y Muntagin, y las subespecies de mayor supervivencia fueron stolonifera y saligna, mientras que la de mejor crecimiento fue la subespecie saligna.

Se sugiere confirmar los resultados con una nueva evaluación a mayor edad de los ensayos y complementarla con análisis de productividad y calidad forrajera, así como también con una evaluación de productividad de semillas, las cuales tendrían importantes aplicaciones potenciales como alimentos funcionales.

Palabras clave: Acacia saligna, procedencias, subespecies, crecimiento, secano Región del Maule

\footnotetext{
${ }^{7}$ Instituto Forestal, Sede Biobío. bgutierr@infor.cl; hsoto@infor.cl

${ }^{8}$ Instituto Forestal, Sede Metropolitana. parojas@infor.cl
} 


\section{SUMMARY}

Acacia saligna is grown as an exotic species in the Coquimbo Region, where there are around 7,500 ha, mainly oriented to forage production, fuel, soil recovery and erosion control. Their seeds would also have potential applications in the functional food industry. The great variability exhibited by the species suggests that it could also be cultivated in the coastal and inland drylands of the Maule region.

Even so, its low digestibility and high tannin contents make A. saligna unattractive forage as a single source of food for livestock. However, this characteristic, as well as its growth, survival and others, can be improved by adequately exploring of the enormous variability that the species presents.

With the aim of analyze the survival and growth of A. saligna, two provenance trials were evaluated in May 2018, one in the transition zone between littoral and dryland of Maule (Chanco) established in 2014 and other one in the interior dryland of the same region (Cauquenes), established in 2015. Both trials represent a collection of 11 provenances of Western Australia that include the four main subspecies recognized for this species: lindleyi, saligna, pruinescens and stolonifera. Before establishing these trials, mainly the subspecies pruinescens and a small participation of lindleyi would have been present in the country.

Through analysis of variance and multiple means comparisons, it is concluded that in the coastal zone (Chanco) the best provenances, in terms of growth and survival, correspond to Bambun Rd. and Palmer Block, while the best subspecies were pruinescens and saligna; on the other hand, in the interior dryland (Cauquenes) the best provenances in terms of survival and growth were Flynn Drive and Muntagin, the subspecies of greater survival were stolonifera and saligna, while the one with the best growth was the subspecies saligna.

It is suggested to confirm the results with a new evaluation at an older age of the trials and to complement it with productivity and forage quality analysis, as well as with an evaluation of seed productivity, which would have important potential applications as functional food.

Key words: Acacia saligna, provenances, subspecies, growth, dryland, Maule Region 


\section{INTRODUCCIÓN}

Acacia saligna es un arbusto de la familia Fabaceae, originario del sudeste de Australia. Es una especie colonizadora, que puede crecer en suelos pobres y sitios con baja precipitación, razón por la que se ha naturalizado o se la cultiva en distintas regiones del mundo. Se planta principalmente en zonas semiáridas, donde constituye una interesante fuente de forraje, leña y taninos, teniendo además usos como cortina cortaviento, estabilización de dunas, protección del suelo, fijación de nitrógeno y ornamento, entre otros.

En Chile la especie es considerada un recurso forestal con aptitud para el establecimiento de sistemas agroforestales en la zona semiárida, donde se han registrado producciones de 0,8 a 2,2 toneladas de forraje seco por hectárea en plantaciones de 3 a 4 años establecidas en el secano interior de la provincia de Chopa (Perret y Mora, 1999), aunque su baja digestibilidad y altos contenidos de taninos hacen de $A$. saligna un forraje poco atractivo como fuente única de alimento para ganado (George et al., 2007).

Por otra parte, la especie posee un interesante potencial para uso agroalimentario mediante la producción de harina a partir de sus semillas. Esta harina posee atractivas características funcionales, entre ellas un alto contenido de componentes antioxidantes (mayores que el maqui y el calafate), alto porcentaje de proteínas, actividad antihipertensiva y presencia de ácido djenkólico, este último tendría actividad como controlador de diabetes tipo 2 (Rojas, 2011; Rojas et al., 2014).

A. saligna se caracteriza por exhibir gran variabilidad morfológica y también en atributos ecológicos y biológicos. Tal variabilidad ha motivado que se diferencien cuatro sub especies o variedades geográficas: (i) $s s p$ saligna conocida como la variedad "cyanophylla"; (ii) $s s p$ pruinescens como la variedad "Tweed River" o "Blue"; (iii) ssp lindleyi como la variedad "típica"; y (iv) ssp stolonifera como la variedad "forestal". Las subespecies muestran importantes diferencias en cuanto a su hábito de crecimiento, adaptabilidad climática, potencial de biomasa (follaje para la producción de forraje y/o semillas), valor nutricional para forraje, capacidad de retoñación y propagación vegetativa (estacas y/o estolones), se diferencian también a nivel molecular y ofrecen una importante fuente de variación para programas de selección y mejoramiento genético (George et al., 2006; 2007; McDonald et al., 2007; Millar et al., 2008; 2011) .

Durante la década de 1970, el Instituto Forestal (INFOR) probó en Chile diferentes especies del género Acacia tolerantes a la sequía, siendo Acacia saligna la especie que mostró mejor adaptación y productividad en las zonas semiáridas del país. Su gran adaptación permitió mediante el DL 701 la plantación de hasta 15.000 hectáreas en la Región de Coquimbo (Cerda, 2007), y otras superficies menores en distintas regiones del país. Posteriormente la existencia de acacia saligna en la región de Coquimbo ha disminuido, estimándose en aproximadamente 7.500 ha (Gonzalez, 2014, cit. por Rojas et al., 2014).

Tras su introducción se han desarrollado experiencias silvícolas y genéticas y establecido diversos ensayos para evaluar técnicas de establecimiento, producción forrajera, efecto de riego, determinación de parámetros genéticos y selección de procedencias para distintas condiciones de sitio. En este último contexto se enmarca el establecimiento de dos ensayos de procedencias y subespecies de Acacia saligna, uno de ellos establecido el año 2014 en la zona de transición entre la costa y el secano costero de la región del Maule (Chanco); y otro en el año 2015, en el secano interior de la misma región (Cauquenes). La principal relevancia de estos ensayos es que serían los primeros que consideran a las 4 subespecies tradicionalmente definidas para $A$. saligna, por cuanto según McDonald et al. (2007), las plantaciones existentes en Chile (región de Coquimbo, donde se concentran sus existencias) corresponderían principalmente a la subespecie pruinescens, con alguna participación de lindleyi en algunos antiguos ensayos de INFOR (Tongoy), en tanto que las subespecies saligna y stolonifera no estarían representadas en las plantaciones existentes.

El objetivo de este artículo es evaluar la supervivencia y el crecimiento de dichos ensayos, en el entendido que la variación en supervivencia y crecimiento constituye la información básica para posteriormente determinar el material más adecuado para fines forrajero y alimentario, que permita generar una alternativa de cultivo forestal de interés para la Región del Maule. 


\section{MATERIAL Y MÉTODO}

\section{Ensayos y Material Genético}

Se evalúan dos ensayos de procedencias y subespecies de Acacia saligna.

- Ensayo Chanco: Corresponde a una prueba de procedencias de $A$. saligna instalada el año 2014 en el predio "El Manzano", de propiedad de un particular en la zona de transición entre el litoral y el secano interior de la comuna de Chanco, provincia de Cauquenes en la región del Maule (Figura $\mathrm{N}^{\circ}$ 1; Cuadro $\mathrm{N}^{\circ} 1$ ). El ensayo se compone de cuatros bloques completos al azar, donde se prueban 11 procedencias australianas de la especie, más un testigo compuesto por una raza local de la misma especie (Cuadro $\mathrm{N}^{\circ} 2$; Figura $\mathrm{N}^{\circ} 2$ ). En cada bloque las procedencias son representadas por parcelas cuadradas de 16 plantas (12 parcelas por bloque), alcanzando un número de 192 plantas por bloque y 768 plantas por el ensayo en su totalidad, con un marco de plantación de $3 \times 4$ metros.

- Ensayo Cauquenes: Corresponde a una prueba de procedencias de Acacia saligna instalada el año 2015 en terrenos de la Estación Experimental Cauquenes de INIA, en el secano interior de la comuna de Cauquenes, en la provincia del mismo nombre en la región del Maule (Figura $\mathrm{N}^{\circ} 1$; Cuadro $\mathrm{N}^{\circ} 1$ ). El ensayo se compone de dos conglomerados de seis bloques cada uno, donde se prueban 11 procedencias australianas de $A$. saligna, más un lote adicional de procedencias mezcladas sin identificar (Cuadro $\mathrm{N}^{\circ} 2$; Figura $\mathrm{N}^{\circ} 2$ ). Adicionalmente se prueba el origen de las plantas, diferenciándose plantas originadas de semilla y plantas obtenidas por vía vegetativa mediante enraizamiento de estacas. En cada bloque, de ambos conglomerados, las procedencias son representadas por parcelas lineales de 5 plantas (15 parcelas en cada bloque del conglomerado 1; y 10 parcelas en cada uno de los bloques del conglomerado 2). En total el ensayo alcanza a 750 plantas (450 en conglomerado 1 y 350 en conglomerado 2), establecidas a un espaciamiento de $3 \times 3$ metros y rodeado por una hilera de aislación perimetral.

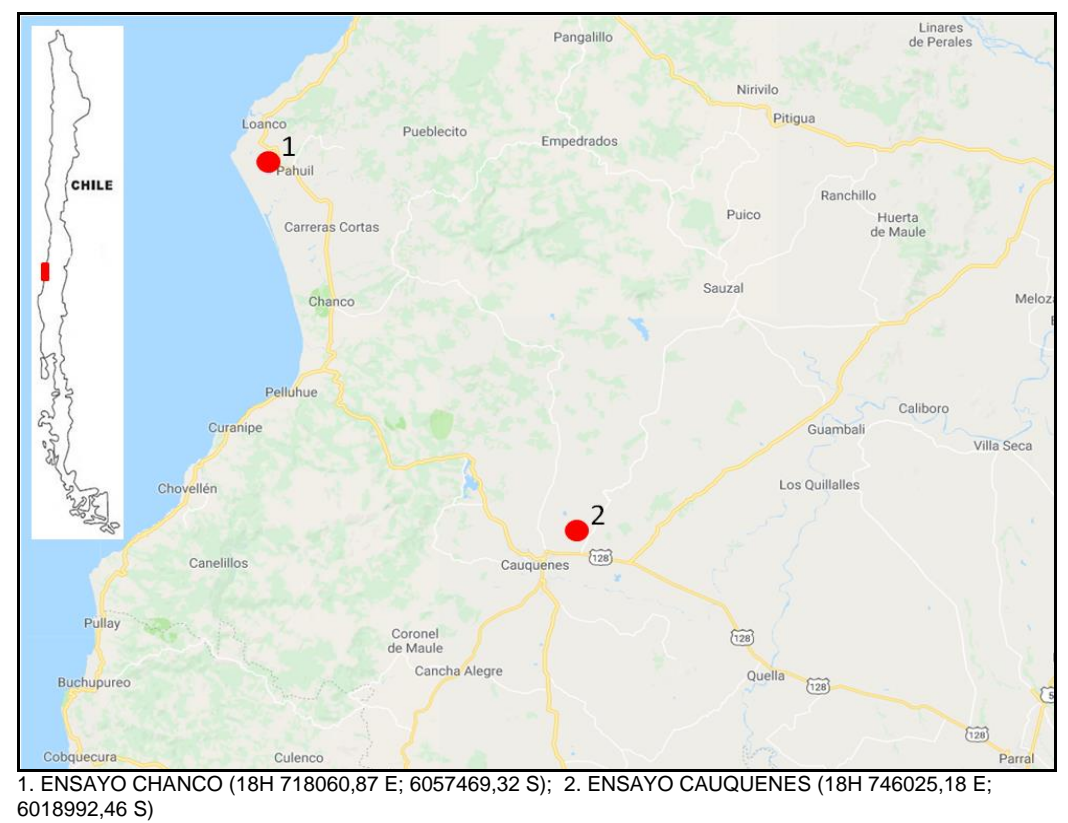

Figura $\mathrm{N}^{\circ} 1$

ENSAYOS DE PROCEDENCIAS DE Acacia saligna 
Cuadro $\mathrm{N}^{\circ} 1$

CARACTERIZACIÓN CLIMÁTICAS DE ZONAS DE EMPLAZAMIENTO DE LOS ENSAYOS

\begin{tabular}{|l|c|c|}
\hline Característica & Ensayo Chanco & Ensayo Cauquenes \\
\hline Ubicación & Litoral-Secano interior & Secano interior \\
\hline $\mathrm{N}^{\circ}$ de zona en estudio (MMA, 2016) & $418-419$ & 416 \\
\hline $\mathrm{T}^{\circ}$ media estival (Dic-Feb) & $16,5^{\circ} \mathrm{C}$ & $18,2^{\circ} \mathrm{C}$ \\
\hline $\mathrm{T}^{\circ}$ media invernal (Jun-Ago) & $9,5^{\circ} \mathrm{C}$ & $9,5^{\circ} \mathrm{C}$ \\
\hline $\mathrm{T}^{\circ}$ máx. media del mes más cálido (Ene) & $23,3^{\circ} \mathrm{C}$ & $26,3^{\circ} \mathrm{C}$ \\
\hline $\mathrm{T}^{\circ}$ min media del mes más frio (Jul) & $6,0^{\circ} \mathrm{C}$ & $6,3^{\circ} \mathrm{C}$ \\
\hline Precipitación anual normal & $845 \mathrm{~mm}$ & $994 \mathrm{~mm}$ \\
\hline
\end{tabular}

(Fuente: MMA, 2016)

Cuadro $\mathrm{N}^{\circ} 2$

MATERIAL GENÉTICO CONSIDERADO EN LOS ENSAYOS DE Acacia saligna DE CHANCO Y CAUQUENES

\begin{tabular}{|c|c|c|c|c|c|}
\hline \multicolumn{2}{|r|}{ Procedencias } & \multirow{2}{*}{\multicolumn{2}{|c|}{ Subespecie (tipo) }} & \multirow{2}{*}{ Lat. S } & \multirow{2}{*}{ Long. E } \\
\hline Código & Nombre & & & & \\
\hline 1 & Arrowsmith & lindleyi & (typical) & $29^{\circ} 36^{\prime \prime}$ & $115^{\circ} 07^{\prime}$ \\
\hline 2 & Bambun Rd & saligna & (cyanophylla) & $31^{\circ} 25^{\prime}$ & $115^{\circ} 53^{\prime}$ \\
\hline 3 & Flynn Drive & saligna & (cyanophylla) & $31^{\circ} 41^{\prime}$ & $115^{\circ} 45^{\prime}$ \\
\hline 4 & Atwood Rd & stolonifera & (forest) & $33^{\circ} 32^{\prime}$ & $115^{\circ} 56^{\prime}$ \\
\hline $5-6$ & Palmer Block & pruinescens & (blue, o tweed river) & $33^{\circ} 15^{\prime}$ & $116^{\circ} 11^{\prime}$ \\
\hline 7 & Mingenew & lindleyi & (typical) & $29^{\circ} 17^{\prime}$ & $115^{\circ} 31^{\prime}$ \\
\hline 8 & Muntagin & lindleyi & (typical) & $31^{\circ} 45^{\prime}$ & $118^{\circ} 34^{\prime}$ \\
\hline 9 & Lake Coolengup & saligna & (cyanophylla) & $32^{\circ} 17^{\prime}$ & $115^{\circ} 47^{\prime}$ \\
\hline 10 & Parkeyeering & lindleyi & (typical) & $33^{\circ} 20^{\prime}$ & $117^{\circ} 21^{\prime}$ \\
\hline 11 & Murchinson River & lindleyi & (typical) & $27^{\circ} 49^{\prime}$ & $114^{\circ} 40^{\prime}$ \\
\hline 12 & Chilean Land Race & \multicolumn{4}{|c|}{ Testigo ensayo Chanco } \\
\hline NN & Mezcla de Procedencias & \multicolumn{4}{|c|}{ Testigo Ensayo Cauquenes } \\
\hline
\end{tabular}

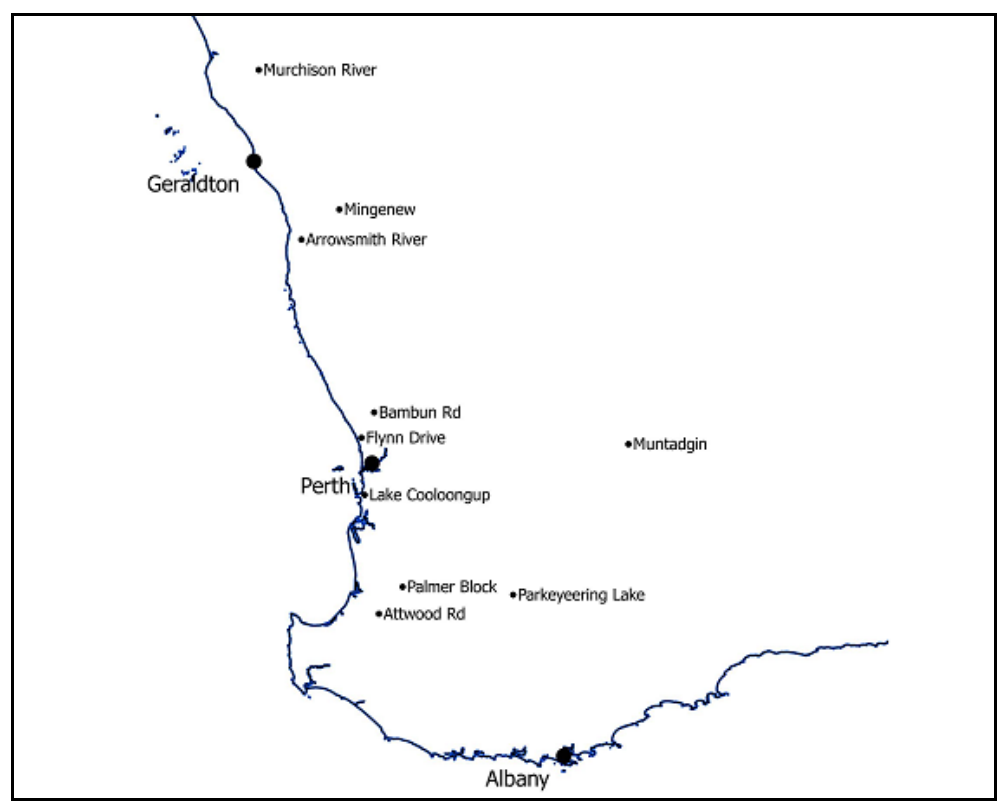

Figura $\mathrm{N}^{\circ} 2$

DISTRIBUCIÓN DE LAS PROCEDENCIAS DE Acacia saligna EN SUROESTE AUSTRALIANO ENSAYADAS EN LA REGIÓN DEL MAULE 
Una síntesis de las características climáticas de las procedencias ensayadas se presenta en el Cuadro $\mathrm{N}^{\circ} 3$. Aspectos de la morfología de las subespecies se presentan en la Figura $\mathrm{N}^{\circ} 3$

\section{Cuadro $\mathrm{N}^{\circ} 3$}

CARACTERIZACIÓN CLIMÁTICA DE PROCEDENCIAS Y SUBESPECIES DE Acacia saligna

\begin{tabular}{|c|c|c|c|c|c|}
\hline Subespecie & Procedencias & $\begin{array}{c}\text { Rango } \\
\text { Altitudinal }\end{array}$ & $\begin{array}{l}\mathrm{T}^{\circ} \text { Media } \\
\text { máx. mes } \\
\text { más cálido }\end{array}$ & $\begin{array}{l}\mathrm{T}^{\circ} \text { Media } \\
\text { min. mes } \\
\text { más frio }\end{array}$ & $\begin{array}{l}\text { Precipitación } \\
\text { Anual }\end{array}$ \\
\hline $\begin{array}{l}\text { lindleyi } \\
\text { (Typical) }\end{array}$ & $\begin{array}{l}\text { 1.Arrowsmith } \\
\text { 7.Mingenew } \\
\text { 8.Muntagin } \\
\text { 10.Parkeyeering } \\
\text { 11.Murchinson River }\end{array}$ & $100-350 \mathrm{msnm}$ & $28-39^{\circ} \mathrm{C}$ & $5-9^{\circ} \mathrm{C}$ & $250-600 \mathrm{~mm}$ \\
\hline $\begin{array}{l}\text { saligna } \\
\text { (Cyanophylla) }\end{array}$ & $\begin{array}{l}\text { 2.Bambun Rd } \\
\text { 3.Flynn Drive } \\
\text { 9.Lake Coolengup }\end{array}$ & 0-90 msnm & $28-33^{\circ} \mathrm{C}$ & $8-10^{\circ} \mathrm{C}$ & $750-900 \mathrm{~mm}$ \\
\hline $\begin{array}{l}\text { pruinescens } \\
\text { (Tweed River o } \\
\text { Blue) }\end{array}$ & 5-6.Palmer Block & $150-300 \mathrm{msnm}$ & $30-31^{\circ} \mathrm{C}$ & $4-6^{\circ} \mathrm{C}$ & $800-1.000 \mathrm{~mm}$ \\
\hline $\begin{array}{l}\text { stolonifera } \\
\text { (forest) }\end{array}$ & 4.Atwood Rd & $5-300 \mathrm{msnm}$ & $27-30^{\circ} \mathrm{C}$ & $6-8^{\circ} \mathrm{C}$ & $900-1.000 \mathrm{~mm}$ \\
\hline
\end{tabular}

(Fuente: http://worldwidewattle.com)
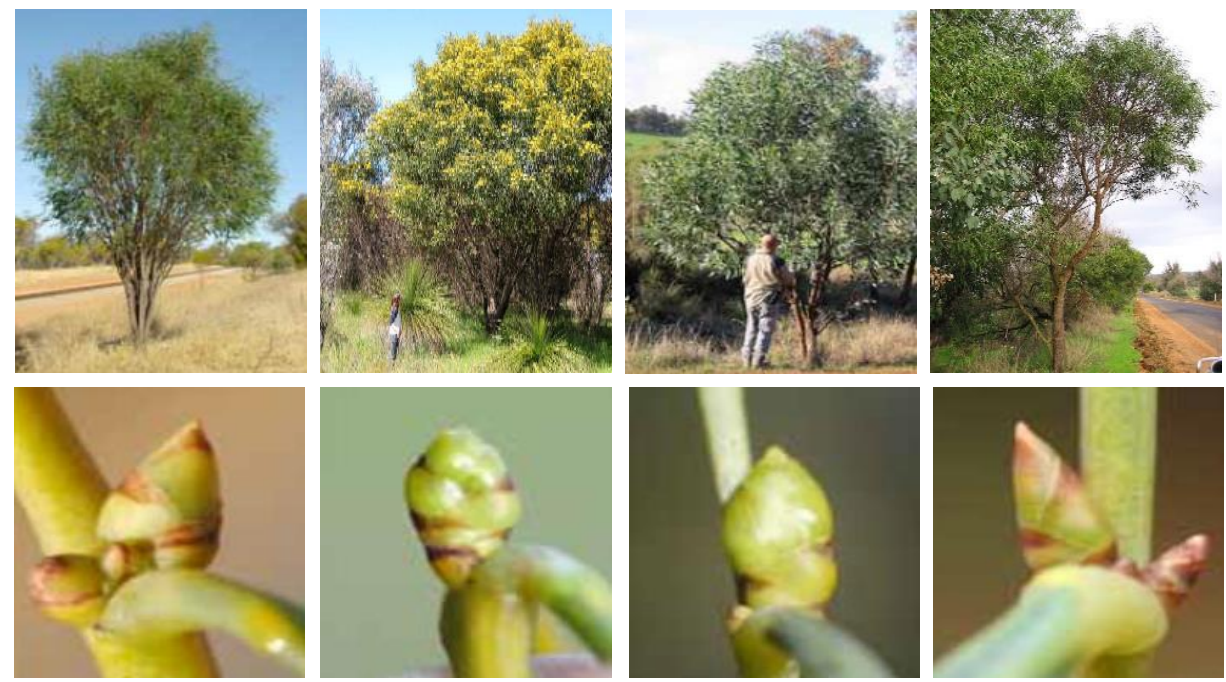

(Fuente: Adaptado de http://worldwidewattle.com y Maslin et al., 2011)

FIGURA $N^{\circ} 3$

HÁBITO DEL ÁRBOL Y ASPECTO DE LAS YEMAS DE INFLORESCENCIAS DE LAS SUBESPECIES DE Acacia saligna (de izquierda a derecha: lindleyi, saligna, pruinescens y stolonifera)

\section{Medición y Evaluación}

Los ensayos de Chanco y Cauquenes fueron medidos a comienzos de mayo de 2018, ocasión en que tenían 4 y 3 años, respectivamente (Figura $\left.N^{\circ} 4\right)$. Haciendo uso de los respectivos croquis se definió una ruta de medición para cada ensayo, la cual fue empleada para construir los formularios de levantamiento de datos. Se definió las variables a medir, que fueron representadas en los formularios correspondientes y fueron las siguientes: 
- Altura total: Medida con vara telescópica (m).

- Número de vástagos desde el cuello (cantidad).

- Diámetro de cada vástago: Medido a la altura del cuello con piedemetro (mm).

- Diámetro de copa: Medido en dos direcciones perpendiculares entre sí (N-S y E-W) (m).

- Madurez reproductiva: Evaluación visual de presencia de órganos reproductivos (1= presentes; $0=$ ausentes)

- Un campo para observaciones complementarias.

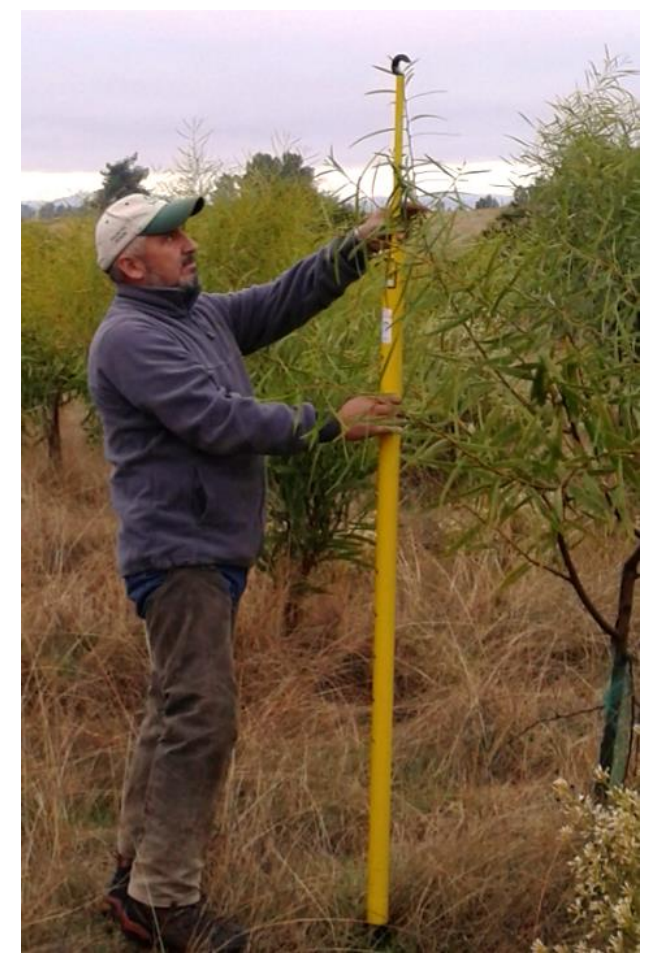

Figura $\mathrm{N}^{\circ} 4$

MEDICIÓN DE ALTURA EN INDIVIDUOS DE Acacia saligna DE ENSAYO CHANCO

Los datos registrados en los formularios de terreno fueron digitados en planillas Excel, conformando las bases de datos con las mediciones de cada ensayo. Mediante histogramas y gráficos de dispersión se validaron los datos verificando la ausencia de errores evidentes de digitación y datos anómalos o fuera de rango.

Posteriormente, con los datos de terreno validados y depurados, se procedió a construir las variables sobre las cuales conducir el análisis y las comparaciones. Para estos efectos se determinó para cada árbol:

DAC equivalente: Correspondiente al diámetro de cuello que genera una superficie equivalente a la suma de las superficies generadas por los diámetros de cuello de cada uno de sus vástagos, expresado en el diámetro de esta superficie $(\mathrm{cm})$. 
Estimador general de biomas $\mathrm{D}^{2} \mathrm{H}$ : Correspondiente al cuadrado del DAC equivalente ponderado por la altura $\left(\mathrm{dm}^{3}\right)$.

Área de copa: Correspondiente a la superficie de proyección circular de copa obtenida a partir de los valores de diámetro de copa registrados en terreno $\left(\mathrm{m}^{2}\right)$.

Esta información se incorporó a la base de datos junto con la información de las subespecies correspondientes a cada procedencia

Se estimó la supervivencia promedio por procedencia y subespecie. Se efectuaron análisis de varianza $(p=0,05)$ y comparaciones múltiples de medias (Scott y Knott) entre procedencias y subespecies para la altura, DAC, $D^{2} \mathrm{H}$ y Área de Copa. Los resultados se tabularon y graficaron para efectos de comparación entre procedencias, subespecies y ensayos.

\section{RESULTADOS Y DISCUSIÓN}

Durante la medición se observó que el ensayo de Cauquenes presenta una muy baja supervivencia (48\%), la que en el conglomerado 1, que constituye el ensayo propiamente tal, apenas alcanza a un $28,7 \%$, observándose también alta incidencia de daño por frio, con plantas de escaso crecimiento y que frecuentemente constituyen rebrotes de plantas severamente dañadas.

La incidencia del daño y mortalidad se relaciona directamente con el relieve del ensayo, concentrándose en las hileras que ocupan las zonas más bajas del conglomerado 1, donde existen numerosas hileras completas de plantas muertas o con pequeños rebrotes emitidos como respuesta al daño sufrido por la planta original.

Debido a estas situaciones que alteran el comportamiento del ensayo, no se consideró hacer una evaluación detallada del mismo, pues sus resultados están severamente alterados por factores ajenos al que se pretende evaluar y que enmascaran o confunden el efecto de las procedencias y subespecies sobre el crecimiento y supervivencia, que es el objetivo de esta evaluación.

Consecuentemente, para el ensayo Cauquenes solo se estimaron parámetros de estadística descriptiva en forma conjunta para los conglomerados 1 y 2 , los que se utilizaron como referencia para discutir y analizar en detalle los resultados del ensayo de Chanco, el que sí presenta supervivencia y crecimiento de mayor interés y menores alteraciones (Cuadro $\mathrm{N}^{\circ} 4$ ).

\section{Cuadro $\mathrm{N}^{\circ} 4$}

COMPARACIÓN DE VALORES TOTALES POR ENSAYO

\begin{tabular}{|l|c|c|c|c|c|c|}
\hline \multirow{2}{*}{ Ensayo } & \multirow{2}{*}{$\mathbf{n}$} & \multirow{2}{*}{\begin{tabular}{c} 
Superv \\
\cline { 4 - 6 }
\end{tabular}} & & \multicolumn{2}{|c|}{ Altura } & \multicolumn{2}{c|}{ DAC } \\
\cline { 4 - 7 } & & $\begin{array}{c}\text { Prom (D.E.) } \\
(\mathbf{m})\end{array}$ & $\begin{array}{c}\text { IMA } \\
(\mathbf{m} / \text { año) }\end{array}$ & $\begin{array}{c}\text { Prom (D.E.) } \\
(\mathbf{c m})\end{array}$ & $\begin{array}{c}\text { IMA } \\
\text { (cm/año) }\end{array}$ \\
\hline Cauquenes (3 años) & $129 / 450$ & 28,7 & $1,76(0,74)$ & 0,59 & $3,98(1,78)$ & 1,33 \\
\hline Chanco (4 años) & $657 / 768$ & 85,5 & $2,48(0,75)$ & 0,62 & $7,92(2,57)$ & 1,98 \\
\hline
\end{tabular}

En términos de incrementos medios anuales los dos ensayos presentan valores similares, en ambos casos los incrementos medios anuales de altura se enmarcan en el intervalo de 0,3 a 0,7 m/año que menciona Rojas (2011) para plantaciones de $A$. saligna irrigadas en la zona norte de Chile (Región de Coquimbo). Sin embargo, las alturas observadas en los ensayos resultan muy inferiores a los $8 \mathrm{~m}$ que según el mismo autor alcanzaría esta especie a los 4 a 5 años de edad en sitios con pocas limitaciones para el crecimiento de las plantas.

Otra referencia para comparar los crecimientos observados en los ensayos de la región del Maule la constituye el estudio de Mora y Meneses (2003), quienes determinaron valores de 
DAC de 1,06 a 2,18 cm para plantas de Acacia saligna de 16 meses de edad, en un ensayo de irrigación establecido en la Región de Coquimbo. En la misma situación determinaron valores de altura de 0,6 a 1,55 m, siendo en ambos casos los valores más bajos los correspondientes al testigo sin riego y los más altos al de plantas que recibieron $8 \mathrm{~L}$ de agua por mes.

\section{Supervivencia y Madurez Reproductiva}

El ensayo de Chanco exhibe una supervivencia promedio de $85,5 \%$ y un $9,1 \%$ de sus individuos exhibe evidencias de haber alcanzado la fase de madurez reproductiva. A nivel de procedencias los resultados se resumen en el Cuadro $\mathrm{N}^{\circ} 5$, donde se observa que Bambun Rd., Palmer Block y Flynn Drive logran los mayores niveles de supervivencia, mientras que Atwood River se destaca por presentar el mayor porcentaje de individuos en fase reproductiva $(43,6 \%)$, seguido por Palmer Block con 22,8\%.

A nivel de subespecies, el Cuadro $N^{\circ} 6$ indica que la mejor $(94,0 \%)$ y peor $(81,9 \%)$ supervivencia corresponden a las subespecies pruinescens y lindleyi, respectivamente. Esta última es la que presenta menor proporción de individuos en fase reproductiva, mientras que la mayor cantidad de árboles en fase reproductiva se presenta en la subespecie stolonifera.

\section{Cuadro $\mathrm{N}^{\circ} 5$}

SUPERVIVENCIA Y MADUREZ REPRODUCTIVA POR PROCEDENCIA EN ENSAYO CHANCO

\begin{tabular}{|c|c|c|c|c|c|}
\hline \multicolumn{2}{|r|}{ Procedencia } & \multirow{2}{*}{ Subespecie (tipo) } & \multirow{2}{*}{$\mathbf{n}$} & \multirow{2}{*}{$\begin{array}{c}\text { Supervivencia } \\
(\%)\end{array}$} & \multirow{2}{*}{$\begin{array}{c}\text { Madurez } \\
\text { Reproductiva } \\
(\%)\end{array}$} \\
\hline Código & Nombre & & & & \\
\hline 2 & Bambun Rd & saligna (cyanophylla) & 62 & 96,9 & 6,5 \\
\hline $5-6$ & Palmer Block & pruinescens (blue) & 79 & 94,0 & 22,8 \\
\hline 3 & Flynn Drive & saligna (cyanophylla) & 15 & 93,8 & 6,7 \\
\hline 7 & Mingenew & lindleyi (typical) & 96 & 93,3 & 3,1 \\
\hline 4 & Atwood Rd & stolonifera (forest) & 39 & 90,9 & 43,6 \\
\hline 12 & Chilean Landrace & & 57 & 89,1 & 19,3 \\
\hline 10 & Parkeyeering & lindleyi (typical) & 90 & 88,5 & 0,0 \\
\hline 1 & Arrowsmith & lindleyi (typical) & 85 & 82,7 & 2,4 \\
\hline 8 & Muntagin & lindleyi (typical) & 50 & 79,7 & 2,0 \\
\hline 9 & Lake Coolengup & saligna (cyanophylla) & 9 & 68,8 & 11,1 \\
\hline 11 & Murchinson River & lindleyi (typical) & 63 & 64,4 & 1,6 \\
\hline \multicolumn{3}{|c|}{ Total Ensayo } & 645 & 85,5 & 9,1 \\
\hline
\end{tabular}

Cuadro $\mathrm{N}^{\circ} 6$

SUPERVIVENCIA Y MADUREZ REPRODUCTIVA POR SUBESPECIE EN ENSAYO CHANCO

\begin{tabular}{|l|r|r|r|}
\hline Subespecie & \multicolumn{1}{|c|}{$\mathbf{n}$} & \multicolumn{1}{c|}{$\begin{array}{c}\text { Supervivencia } \\
(\%)\end{array}$} & $\begin{array}{c}\text { Madurez } \\
\text { Reproductiva } \\
(\%)\end{array}$ \\
\hline pruinescens (blue) & 79 & 94,0 & 22,8 \\
\hline saligna (cyanophylla) & 86 & 91,7 & 7 \\
\hline stolonifera (forest) & 39 & 90,9 & 43,6 \\
\hline Chilean landrace & 57 & 89,1 & 19,3 \\
\hline lindleyi (typical) & 384 & 81,9 & 1,8 \\
\hline Total ensayo & $\mathbf{6 4 5}$ & $\mathbf{8 5 , 5}$ & $\mathbf{9 , 1}$ \\
\hline
\end{tabular}




\section{Variables de Crecimiento}

Existen diferencias estadísticamente significativas de crecimiento entre procedencias. El mismo fenómeno fue descrito por Mora y Meneses (2004) para dos ensayos de procedencias de Acacia saligna evaluado a los 15 meses de edad en la Región de Coquimbo.

En el ensayo de Chanco las procedencias Palmer Block, Bambun Rd. y Lake Coolengup consistentemente conforman un grupo que alcanza el mayor desarrollo en altura, diámetro de cuello, $\mathrm{D}^{2} \mathrm{H}$ y superficie de copa (Cuadro $\mathrm{N}^{\circ} 7$ ), las dos primeras son las mismas que logran la mayor supervivencia, mientras que la tercera resulta ser la de peor supervivencia entre las procedencias ensayadas, situación que hace menos interesante su destacado crecimiento.

En términos comparativos el crecimiento en altura a los cuatro años registrado en Chanco, con valores medios por procedencia de entre 2,5 y 2,9 m, no parece considerablemente superior al obtenido por la misma especie en las condiciones más adversas de la Región de Coquimbo, donde a los 15 meses de edad alcanzó valores medios por procedencia de entre 0,6 y 1,6 m (Mora y Meneses, 2004).

Por el contrario, en términos de diámetro de cuello los valores medios por procedencia, de 6,3 a 9,5 cm a los cuatro años en Chanco, parecen superiores a los observados en Coquimbo a los 15 meses de 1,3 a $2,1 \mathrm{~cm}$.

Cabe destacar que los resultados obtenidos por las mejores procedencias de los ensayos de Coquimbo son particularmente sobresalientes y de la misma magnitud que los logrados por plantas regadas con $8 \mathrm{~L}$ de agua por mes, a los 15 meses de edad; análogamente las peores procedencias obtienen resultados similares a los de plantas sin regar establecidas en Coquimbo (Cuadro $\mathrm{N}^{\circ} 8$ )

Cuadro $\mathrm{N}^{\circ} 7$

VARIABLES DE CRECIMIENTO POR PROCEDENCIAS EN ENSAYO CHANCO

\begin{tabular}{|c|c|c|c|c|c|c|c|c|}
\hline \multicolumn{2}{|c|}{ Procedencia } & \multirow{2}{*}{ Subespecie (tipo) } & \multirow{2}{*}{$\mathbf{n}$} & \multirow{2}{*}{$\begin{array}{c}\text { Altura } \\
(\mathrm{m})\end{array}$} & \multirow{2}{*}{$\begin{array}{l}\text { DAC } \\
(\mathrm{cm})\end{array}$} & \multirow{2}{*}{$\begin{array}{l}\mathrm{D}^{2} \mathrm{H} \\
\left(\mathrm{dm}^{3}\right)\end{array}$} & \multirow{2}{*}{\multicolumn{2}{|c|}{$\begin{array}{c}\text { SUP COPA } \\
\left(\mathrm{m}^{2}\right)\end{array}$}} \\
\hline Código & Nombre & & & & & & & \\
\hline $5-6$ & Palmer Block & pruinescens (blue) & 79 & 2,89 & 9,51 & 30,21 & 7,18 & a \\
\hline 2 & Bambun Rd & saligna (cyanophylla) & 62 & 2,97 & 9,61 & 30,10 & 7,41 & a \\
\hline 9 & Lake Coolengup & saligna (cyanophylla) & 9 & 2,99 & 9,19 & 28,82 & 10,04 & $a$ \\
\hline 8 & Muntagin & lindleyi (typical) & 50 & 2,75 & 8,08 & 21,52 & 6,33 & $b$ \\
\hline 3 & Flynn Drive & saligna (cyanophylla) & 15 & 2,65 & 8,63 & 21,23 & 6,77 & $\mathrm{~b}$ \\
\hline 12 & Chilean landrace & & 57 & 2,36 & 8,47 & 20,24 & 6,25 & $\mathrm{~b}$ \\
\hline 4 & Atwood Rd & stolonifera (forest) & 39 & 2,26 & 7,89 & 17,75 & 4,96 & c \\
\hline 1 & Arrowsmith & lindleyi (typical) & 85 & 2,28 & 7,50 & 16,30 & 7,03 & a \\
\hline 11 & Murchinson River & lindleyi (typical) & 63 & 2,15 & 7,18 & 14,32 & 7,81 & $\mathrm{a}$ \\
\hline 7 & Mingenew & lindleyi (typical) & 96 & 2,07 & 7,13 & 13,90 & 6,10 & $b$ \\
\hline 10 & Parkeyeering & lindleyi (typical) & 90 & 2,53 & 6,29 & 12,32 & 3,27 & $d$ \\
\hline \multicolumn{3}{|c|}{ Total ensayo } & 645 & 2,48 & 7,92 & 19,45 & 6,32 & \\
\hline
\end{tabular}


Cuadro $\mathrm{N}^{\circ} 8$

COMPARACIÓN DE CRECIMIENTOS EN ALTURA Y DAC DE Acacia saligna

EN ENSAYO CHANCO CON ENSAYOS DE REFERENCIA

\begin{tabular}{|l|c|c|c|}
\hline Ensayo & Edad & $\begin{array}{c}\text { Altura } \\
(\mathbf{m})\end{array}$ & $\begin{array}{c}\text { DAC } \\
(\mathbf{c m})\end{array}$ \\
\hline Chanco (Maule) (mejor y peor procedencia) & 4 años & $2,5-2,9$ & $6,3-9,5$ \\
\hline Cauquenes (Maule) (mejor y peor procedencia) & 3 años & $1,3-2,5$ & $2,6-5,9$ \\
\hline El Tangue (Coquimbo) (mejor y peor procedencia) & 15 meses & $0,6-1,1$ & $1,5-2,1$ \\
\hline Cuz Cuz (Coquimbo) (mejor y peor procedencia) & 15 meses & $0,7-1,6$ & $1,3-2,4$ \\
\hline $\begin{array}{l}\text { Higueritas Unidas (Coquimbo) (testigo sin riego y mejor tratamiento } \\
\text { de 8 L de agua/mes) }\end{array}$ & 16 meses & $0,6-1,6$ & $1,1-2,2$ \\
\hline
\end{tabular}

A nivel de subespecies, se observa una situación similar a la registrada con la supervivencia, siendo pruinescens la subespecie de mejor desempeño en todas las variables de crecimiento, sin diferenciarse significativamente de saligna. Por su parte lindleyi además de exhibir la supervivencia más baja, también exhibe los menores valores de crecimiento (Cuadro $N^{\circ} 9$ ).

\section{VARIABLES DE CRECIMIENTO POR SUBESPECIE EN ENSAYO CHANCO}

\begin{tabular}{|l|c|cc|cc|cc|cc|}
\hline Subespecie & $\mathbf{n}$ & \multicolumn{2}{|c|}{$\begin{array}{c}\text { Altura } \\
(\mathbf{m})\end{array}$} & \multicolumn{2}{|c|}{$\begin{array}{c}\text { DAC } \\
(\mathbf{c m})\end{array}$} & \multicolumn{2}{|c|}{$\begin{array}{c}\mathbf{D}^{2} \mathbf{H} \\
\left(\mathbf{d m}^{3}\right)\end{array}$} & $\begin{array}{c}\text { SUP COPA } \\
\left(\mathbf{m}^{2}\right)\end{array}$ \\
\hline pruinescens (blue) & 79 & 2,90 & $\mathrm{a}$ & 9,51 & $\mathrm{a}$ & 30,21 & $\mathrm{a}$ & 7,19 & $\mathrm{a}$ \\
\hline saligna (cyanophylla) & 86 & 2,92 & $\mathrm{a}$ & 9,39 & $\mathrm{a}$ & 28,41 & $\mathrm{a}$ & 7,56 & $\mathrm{a}$ \\
\hline Chilean landrace & 57 & 2,36 & $\mathrm{~b}$ & 8,47 & $\mathrm{~b}$ & 20,25 & $\mathrm{~b}$ & 6,27 & $\mathrm{~b}$ \\
\hline stolonifera (forest) & 39 & 2,26 & $\mathrm{~b}$ & 7,89 & $\mathrm{c}$ & 17,75 & $\mathrm{~b}$ & 5,01 & $\mathrm{~b}$ \\
\hline lindleyi (typical) & 384 & 2,33 & $\mathrm{~b}$ & 7,15 & $\mathrm{c}$ & 15,14 & $\mathrm{c}$ & 5,96 & $\mathrm{~b}$ \\
\hline Total ensayo & $\mathbf{6 4 5}$ & $\mathbf{2 , 4 8}$ & & $\mathbf{7 , 9 2}$ & & $\mathbf{1 9 , 4 5}$ & & $\mathbf{6 , 3 2}$ & \\
\hline
\end{tabular}

Las diferencias de desempeño en crecimiento y otras variables que se observan tanto a nivel de procedencias como de subespecies, obedecen en gran medida a que Acacia saligna exhibe una gran variación en atributos morfológicos, ecológicos y biológicos a lo largo de su distribución natural.

En efecto, la variación es tal, que más que una especie, a Acacia saligna se le considera un complejo taxonómico aún no resuelto (George et al., 2006; 2007; Millar et al., 2011), en el que informalmente se reconocen cuatro subespecies o variantes morfológicas con distintas áreas de distribución ${ }^{9}$.

Estas cuatro entidades son morfológicamente distintas y además presentan un alto grado de divergencia genética que permite conformar tres grupos claramente diferenciados (lindleyi, stolonifera y saligna-pruinescens) (Millar et al., 2008; 2011), situación que explica las diferencias observadas en el ensayo evaluado.

Respecto del mejor desempeño detectado de las subespecies pruinescens y saligna, estas parecen conformar un grupo genético poco diferenciado entre si y diferente al de las otras subespecies.

\footnotetext{
${ }^{9}$ Una quinta subespecie, osullivaniana, ha sido definida en una distribución bien acotada en el norte de Perth, Australia (Millar et al., 2008). McDonald et al. (2007) se refiere a esta quinta subespecie como "cantabilling"
} 
En efecto, George et al. (2006) no pudo diferenciar genéticamente a pruinescens de saligna usando marcadores genéticos RFLP, de modo que solo pudo conformar tres grupos distintos: lindleyi, stolonifera y la combinación saligna-pruinescens.

Por su parte, Millar et al. (2008) indican que ambas subespecies muestran un grado de afinidad genética que también dificulta diferenciarlas con marcadores de microsatélites, no obstante ambas subespecies tienen claras diferencias morfológicas en campo, poseen un área de distribución que no se traslapa y no existe evidencia de hibridación entre sus poblaciones naturales.

Coincidiendo con el mejor crecimiento dasométrico exhibido por las subespecies pruinescens y saligna en el ensayo Chanco, George et al. (2007) observan que estas mismas entidades forman un grupo indistinto y que se diferencia significativamente respecto a las otras subespecies en lo que se refiere a valor forrajero, exhibiendo los mayores niveles de digestibilidad de materia seca, contenido de proteína cruda y contenido de fósforo.

La calidad forrajera varía sustancialmente entre subespecies evidenciando en alguna medida el origen genético de esta variación, así, en la medida en que esta resulte adecuadamente heredable podría ser aumentada mediante mejoramiento genético, permitiendo obtener material de las subespecies pruinescens y saligna que ofrezcan simultáneamente el mejor crecimiento dasométrico, la mayor supervivencia y la mayor calidad forrajera.

Esta perspectiva resulta particularmente interesante, por cuanto abre la posibilidad de explorar y aprovechar la variabilidad genética de la subespecie saligna, la cual según McDonald et al. (2007) no habría estado presente en Chile antes del establecimiento de los ensayos que se evalúan en este artículo.

\section{Interacción con el Sitio}

Cuando la respuesta relativa de las procedencias, $u$ otras entidades genéticas, difiere al crecer en distintos sitios, se está en presencia de interacción genotipo ambiente.

Los gráficos de la Figura $N^{\circ} 5$ permiten contrastar la respuesta en supervivencia y crecimiento exhibido por las procedencias y sub-especies de Acacia saligna en dos condiciones de sitio diferentes, Chanco y Cauquenes, observándose evidencias claras de la existencia de este efecto, el que queda de manifiesto en el hecho de que las mejores procedencias y subespecies no son las mismas en ambos sitios.

La evidencia de interacción genotipo ambiente es coincidente con lo indicado por Mora et al. (2010), quienes en una evaluación cuantitativa específica efectuada a los cinco años de edad en dos ensayos de progenie-procedencia de $A$. saligna establecidos en el norte chico, concluyen la existencia de una significativa interacción para las características de supervivencia, diámetro de copa y altura.

Por el contrario, en lo que se refiere a variables que definen la calidad forrajera de $A$. saligna, George et al. (2007) indican que estas generalmente exhiben una baja interacción genotipo ambiente.

En términos prácticos la incidencia de la interacción genotipo ambiente determina que, para efectos de mejorar crecimiento, se deberá seleccionar material genético específico para cada sitio, por cuanto las mejores procedencias y subespecies identificada en Chanco no necesariamente serán las más apropiadas para cultivarlas en otros sectores con condiciones de sitio diferentes. 

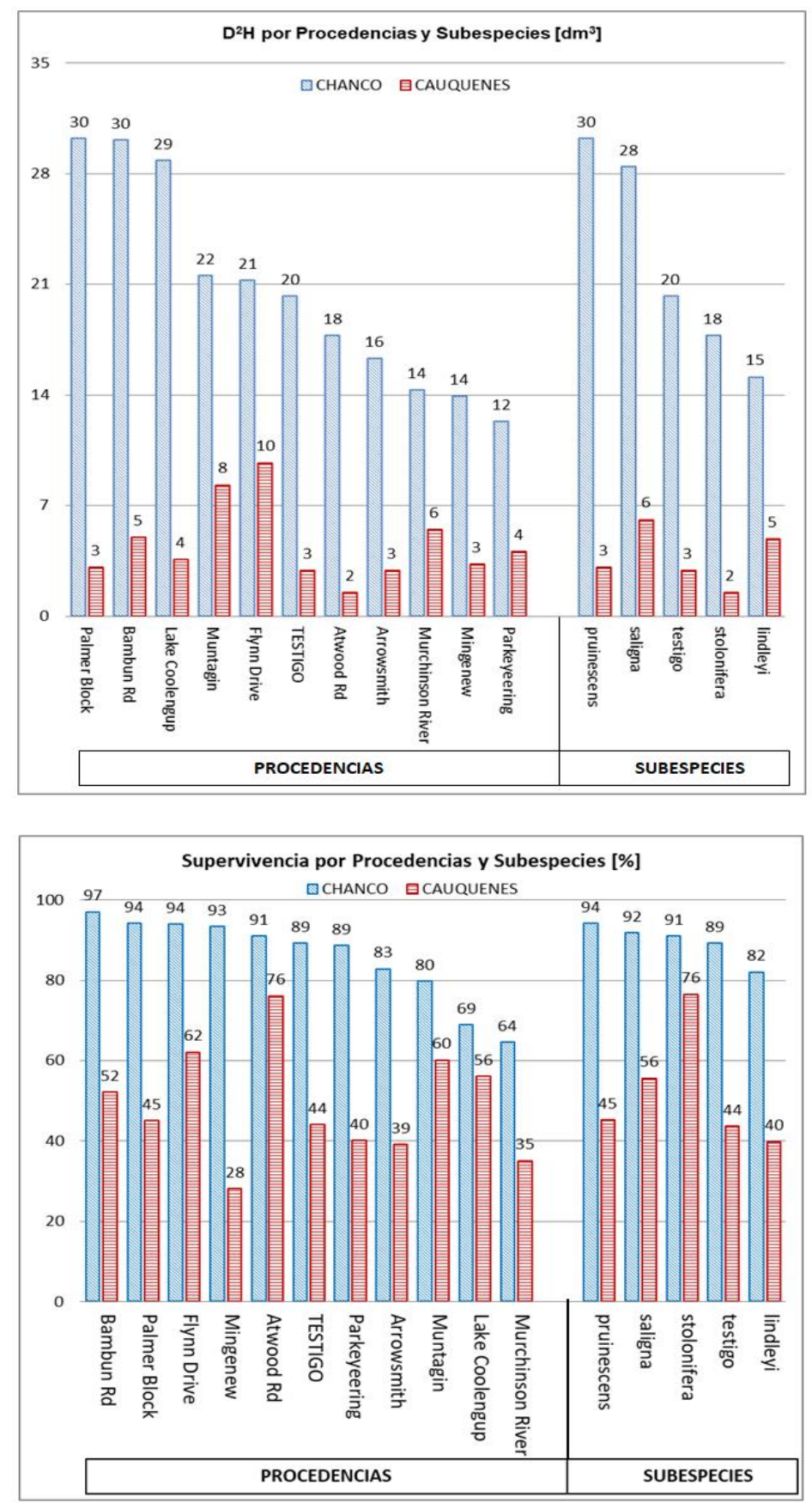

Figura $\mathrm{N}^{\circ} 5$

COMPARACIÓN DE CRECIMIENTO Y SUPERVIVENCIA DE PROCEDENCIAS Y SUBESPECIES DE Acacia saligna EN DOS SITIOS DISTINTOS (CHANCO Y CAUQUENES) 


\section{CONCLUSIONES}

Acacia saligna es un complejo taxonómico de subespecies y tipos geográficos que exhibe una gran variabilidad, la que se refleja en los atributos de crecimiento analizados en este documento y también en diversos otros caracteres señalados en la bibliografía. Esta gran variabilidad explica la expansión de su cultivo como recurso multipropósito en zonas semiáridas de distintas partes del mundo, a la vez que constituye un aspecto básico para mejorar los atributos de interés mediante selección y mejora genética tradicional.

Para condiciones de sitio equivalentes a las del lugar de emplazamiento del ensayo analizado (Chanco, secano costero del Maule), las procedencias de Palmer Block y Bambun Rd. resultan ser las que exhiben el mejor crecimiento y supervivencia. A nivel de subespecies, en las mismas condiciones, pruinescens y saligna se diferencian favorablemente en esos mismos atributos respecto de las subespecies restantes.

Existe evidencia de interacción genotipo-ambiente, que sugiere que en sitios distintos a Chanco el material más apropiado sería diferente al identificado en este sector. Datos referenciales de un ensayo en Cauquenes indican que las mejores procedencias, en cuanto a supervivencia y crecimiento, en el secano interior del Maule serían Flynn Drive y Muntagin. En estas mismas condiciones, las subespecies de mayor supervivencia serían stolonifera y saligna, mientras que la de mejor crecimiento sería la subespecie saligna.

La variabilidad exhibida por la especie es una condición favorable para efectuar su mejoramiento genético, particularmente el promisorio desempeño exhibido por la subespecie saligna en ambos sitios y por stolonifera en el secano interior resultan de especial interés, por cuanto ellas no habrían sido probadas anteriormente en el país, en cuyas plantaciones ha predominado fundamentalmente solo la subespecie pruinescens.

El análisis efectuado en este documento contribuye a identificar el material idóneo para el cultivo de Acacia saligna en el Maule, sugiriéndose confirmarlo con una nueva evaluación a mayor edad de los ensayos y complementarlo con análisis de productividad y calidad forrajera, así como también con una evaluación de productividad de semillas, las cuales tendrían importantes aplicaciones potenciales como alimentos funcionales.

\section{AGRADECIMIENTOS}

Los autores agradecen al personal de INFOR, Sres. Arnoldo Villarroel, Mauricio Navarrete y Jorge Acevedo por su activa participación en el establecimiento y mantención de los ensayos objetos de esta evaluación; del mismo modo, al Sr. Alejandro Lucero, investigador de INFOR, por el suministro de los planos originales y coordinación de apoyo logístico para la faena de medición de los ensayos evaluados en este artículo.

\section{REFERENCIAS}

Cerda, J., 2007. Reseña y futuro del sector forestal en la Región de Coquimbo. Ciencia e Investigación Forestal. Número Extraordinario 13:47-58.

George, N.; Henry, D.; Yan, G. and Byrne, M., 2007. Variability in feed quality between populations of Acacia saligna (Labill) H. Wendl. (Mimosoideae)-Implications for domestication. Animal Feed Science and technology 136(2007): 109-127.

George, N.; Byrne, M.; Maslin, B. and Yan, G., 2006. Genetic differentiation among morphological variants of Acacia saligna (Mimosaceae). Tree Genetics and Genomes (2006 2: 109-119

Maslin, B.; O'Sullivan, W. and McDonald, M., 2011. Understanding Acacia saligna. En: Francis, R. (Ed). Proceedings of the Wattle We Eat for Dinner. Workshop on Australian for Food security. Alice Spring, Australia, 16-18 August 2011. Pp: 21-29.

McDonald, M.; Mazanec, R.; Bartle, J. and Maslim, B., 2007. Improved prospect for the domestication of Acacia 
saligna in region of Coquimbo, Chile. Ciencia e Investigación Forestal, Vol 13, número extraordinario noviembre 2007, Pp: 91-101.

Millar, M.; Byrne, M.; Nuberg, I. and Sedgley, M., 2008. A rapid PCR based diagnostic test for the identification of subspecies of Acacia saligna. Tree Genetics and Genomes 4(4):625-635.

Millar, M.; Byrne, M.; O'Sullivan, W., 2011. Defining entities in the Acacia saligna (Fabaceae) species complex using a population genetics approach. Australian Journal of Botany (2011) 59:137-148.

MMA, 2016. Elaboración de una base digital del clima comunal de Chile: Línea base (1980-2010) y proyección al año 2050. Ministerio de Medio Ambiente, Chile. Información para de Desarrollo Productivo Ltda. Informe Final. Santiago 16 de Junio de 2016. $142 \mathrm{p}$.

Mora, F.; Perret, S.; Scapim, C. and Arnhold, E., 2010. Genetic parameters of growth and survival in Acacia saligna shrubs. Cien. Inv. Agr. 37(2):5-14.

Mora, F. y Meneses, R., 2004. Comportamiento de procedencias de Acacia saligna (Labill.) H.L. Wendl. en la Región de Coquimbo, Chile. Ciencia Florestal 14(1):103-109.

Mora, F. y Meneses, R., 2003. Efecto del riego sobre el crecimiento y producción forrajera de Acacia saligna (Labill) H. Wendl. en la zona árida de Chile. Nota Técnica. Lavras 9(2):255-260.

Perret, S. y Mora, F., 1999. Acacia saligna: Su impacto en el Norte Chico. Revista Chile Forestal, v.274, p.34-36.

Rojas, P.; Jiménez, P. y Quitral, V., 2014. Acacia saligna en Chile: semillas con potencial alimentario funcional. Ciencia e Investigación Forestal 20(1):69-75.

Rojas, P., 2011. Results of Acacia saligna growing in Chile and explorations of new foods products. En: Francis, R. (Ed). Proceedings of the Wattle We Eat for Dinner. Workshop on Australian for Food Security. Alice Spring, Australia, 16-18 August 2011. Pp: 87-97. 
